\title{
COMPLETE MONOTONICITY OF MODIFIED BESSEL FUNCTIONS
}

\author{
MOURAD E. H. ISMAIL
}

(Communicated by Kenneth R. Meyer)

\begin{abstract}
We prove that if $\nu>1 / 2$, then $2^{\nu-1} \Gamma(\nu) /\left[x^{\nu / 2} e^{\sqrt{x}} K_{\nu}(\sqrt{x})\right]$ is the Laplace transform of a selfdecomposable probability distribution while $2^{\nu} \Gamma(\nu+1) x^{-\nu / 2} e^{-\sqrt{x}} I_{\nu}(\sqrt{x})$ is the Laplace transform of an infinitely divisible distribution. The former result is used to show that an estimate of $M$. Wong [13] is sharp. We also prove that the roots of the equations

$$
b^{3} l_{\nu-1}(a \sqrt{z}) / I_{\nu}(a \sqrt{z})=a^{3} I_{\nu-1}(b \sqrt{z}) / I_{\nu}(b \sqrt{z}),
$$

and

$$
b^{3} K_{\nu+1}(a \sqrt{z}) / K_{\nu}(a \sqrt{z})=a^{3} K_{\nu+1}(b \sqrt{z}) / K_{\nu}(b \sqrt{z}), \quad \nu>0, z \neq 0,
$$

lie in a certain sector contained in the open left half plane. This proves and extends a conjecture of $\mathrm{H}$. Hattori arising from his work in partial differential equations.
\end{abstract}

\section{INTRODUCTION}

A probability measure $d \mu$ is infinitely divisible if for every positive integer $n$ there is a probability measure $d \mu_{n}$ such that $d \mu$ is an $n$-fold convolution of $d \mu_{n}$, i.e., $d \mu=d \mu_{n} * d \mu_{n} * \cdots * d \mu_{n}$ [2]. A function $f$ defined on $(0, \infty)$ is said to be completely monotonic (on $(0, \infty)$ ) if $f$ has continuous derivatives of all orders and $f^{(n)}(x)>0, x>0$. Bernstein's theorem [2, 12] characterizes completely monotonic functions as Laplace transforms of positive measures whose support is contained in $[0, \infty)$. When a probability measure $d \mu$ is supported on a subset of $[0, \infty)$ then $d \mu$ is infinitely divisible if and only if its Laplace transform satisfies the conditions

$$
\int_{0}^{\infty} e^{-x t} d \mu(t)=e^{-h(x)}, \quad h(0)=0, \quad \text { and } h^{\prime}(x) \text { is completely monotonic } .
$$

When there is a positive measure $d \omega$ such that $h^{\prime}$ has the representation

$$
h^{\prime}(x)=\int_{0}^{\infty} \frac{d \omega(t)}{x+t}, \quad x>0,
$$

Received by the editors September 5, 1988.

1980 Mathematics Subject Classification (1985 Revision). Primary 33A45; Secondary 35S05, 60E05.

Partially supported by a grant from the National Science Foundation. 
then $\mu$ is selfdecomposable [2]. Selfdecomposable functions are infinitely divisible. A probability distribution satisfying (1.1) and (1.2) is called a generalized gamma convolution. Generalized gamma convolutions form an interesting subclass of the selfdecomposable or infinitely divisible distributions [10].

The notation and terminology in this work follow that of Erdelyi, et al. [1], Feller [2], Watson [11] and Widder [12].

The Laplace transforms of probability measures are usually transcendental special functions so $h^{\prime}, h$ is as in (1.1), is a quotient of two special functions. This led several authors to study the complete monotonicity of various quotients of special functions and of the logarithmic derivatives of solutions of differential and difference equations $[4,5]$. In order to prove the infinite divisibility of the student $t$-distribution it was sufficient to prove that

$$
2^{1-\nu} x^{\nu / 2} K_{\nu}(\sqrt{x}) / \Gamma(\nu)=e^{-h(x)}, \quad \nu>-1 / 2,
$$

and $h^{\prime}$ is completely monotonic, where $K_{\nu}$ is a modified Bessel function. The complete monotonicity of $h^{\prime}$ in the above formula proves the complete monotonicity of $x^{\nu / 2} K_{\nu}(\sqrt{x})$. It is clear that the reciprocal of a nonconstant completely monotonic function is not completely monotonic, hence $x^{-\nu / 2} / K_{\nu}(\sqrt{x})$ is not completely monotonic. However one can slightly modify the latter function and obtain a completely monotonic function. The first main result of this work is the following theorem.

Theorem 1. Let

$$
F_{1}(x, \nu)=2^{\nu-1} \Gamma(\nu) x^{-\nu / 2} e^{-\sqrt{x}} / K_{\nu}(\sqrt{x}) .
$$

The function $F_{1}(x, \nu)$ (respectively $1 / F_{1}(x, \nu)$ ) is the Laplace transform of a generalized gamma convolution when $\nu>1 / 2 \quad(0<\nu<1 / 2)$.

We stated Theorem 1 only for $\nu>1 / 2$ or $\nu<1 / 2$ because $F_{1}(x, 1 / 2)=1$ $[1,(7.2 .40)]$. Theorem 1 shows that $F_{1}(x, \nu)$ is a decreasing function of $x$ on $(0, \infty)$ when $\nu>1 / 2$. Thus we obtain the lower bound

$$
x^{\nu} K_{\nu}(x) e^{x}>2^{\nu-1} \Gamma(\nu), \quad x>0, \nu>1 / 2 .
$$

Note that the left-hand side of (1.4) tends to $2^{\nu-1} \Gamma(\nu)$ as $x \rightarrow 0$, hence the lower bound in (1.4) is the best possible constant lower bound.

Results concerning monotonicity of modified Bessel functions $K_{\nu}$ invariably have companion results involving $I_{\nu}$. A companion to Theorem 1 is

Theorem 2. When $\nu>1 / 2$ the function

$$
F_{2}(x ; \nu):=2^{\nu} \Gamma(\nu+1) x^{-\nu / 2} I_{\nu}(\sqrt{x}) e^{-\sqrt{x}},
$$

is the Laplace transform of an infinitely divisible distribution which is not a generalized gamma convolution. When $0<\nu<1 / 2$ the same conclusion holds for $1 / F_{2}(x, \nu)$.

Theorems 1 and 2 will be proved in $\S 2$. In $\S 3$ we discuss the estimates obtained in Wong [13]. We also give a different proof of Wong's estimates and 
use the lower bound (1.4) to show that these estimates are best possible. Wong used his estimates to obtain an upper bound for the number of eigenvalues below the essential spectrum of a pseudo-differential operator in relativistic quantum mechanics [13].

In $\S 4$ we will prove Theorems 3 and 5 . Theorem 3 will be stated below and Theorem 5 will be stated in $\S 4$.

Theorem 3. Let $a>0, b>0, \nu>0$. When $a \neq b$, the nonreal roots of the transcendental equation

$$
b^{3} I_{\nu-1}(a \sqrt{z}) / I_{\nu}(a \sqrt{z})=a^{3} I_{\nu-1}(b \sqrt{z}) / I_{\nu}(b \sqrt{z}), \quad z \neq 0,
$$

are finitely many and lie inside the circle

$$
\left|z+\frac{\left(a^{2}+b^{2}\right) j_{\nu, k}^{2}}{a^{4}+a^{2} b^{2}+b^{4}}\right|=\frac{a b}{a^{4}+a^{2} b^{2}+b^{4}} j_{\nu, k}^{2},
$$

where $j_{\nu, k}$ is a certain positive zero of the Bessel function $J_{\nu}(z)$. Furthermore the equation (1.6) has infinitely many real roots and they are all negative.

Theorem 3 was motivated by a conjecture of $H$. Hattori [6]. Hattori showed that the eigenvalues of a partial differential operator he was studying are the roots of

$$
b^{3} \operatorname{coth}(a \sqrt{z})=a^{3} \operatorname{coth}(b \sqrt{z}), \quad z \neq 0 .
$$

He then conjectured the existence of a positive number $c$ such that if $z=x+i y$ satisfies (1.8) then $x<0$ and $|y|+c x<0$. The transcendental equation (1.8) is the special case $\nu=1 / 2$ of (1.6). It is easy to confirm Hattori's conjecture from Theorem 3. Indeed we find

$$
c=a b\left(a^{4}+b^{4}+a^{2} b^{2}\right)^{-1 / 2}
$$

since the circle (1.7) lies in the left half plane, does not pass through the origin and the slopes of the tangent lines drawn from the origin to the circle (1.7) are

$$
\pm a b\left(a^{4}+b^{4}+a^{2} b^{2}\right)^{-1 / 2} \text {. }
$$

Thus Theorem 3 gives a numerical value for the conjectured constant $c$. Theorem 3 also generalizes Hattori's conjecture and extends it to Bessel functions.

Theorem 3 has a companion result for the modified Bessel functions $K_{\nu}(z)$. The companion result is Theorem 5 which will be stated and proved in $\S 4$.

Ismail and Kelker [8] introduced certain probability distributions involving modified Bessel functions. They were motivated by problems involving special functions. Ismail and Kelker established the infinite divisibility of the distributions they introduced. Later Pitman and Yor [9] provided a very interesting 
probabilistic set up for the aforementioned distributions. The probability distributions discussed in this note also arise naturally in the theory of Bessel functions and we hope they will also arise in an equally natural way in probability theory.

\section{Proofs of TheOREMS 1 AND 2}

Our proofs of Theorems 1 and 2 use the integral representation

$$
\frac{K_{\nu-1}(\sqrt{x})}{\sqrt{x} K_{\nu}(\sqrt{x})}=\frac{4}{\pi^{2}} \int_{0}^{\infty} \frac{t^{-1} d t}{\left(x+t^{2}\right)\left[J_{\nu}^{2}(t)+Y_{\nu}^{2}(t)\right]}, \quad x>0, \nu \geq 0 .
$$

The above integral representation is implicit in Grosswald [3] and has been put in the form (2.1) by the present author [7]. It was also used in [8]. The analogue of (2.1) for the $I_{\nu}$ functions is the Mittag-Leffler expansion [1, (7.9.3), (7.2.12)]

$$
I_{\nu+1}(x) / I_{\nu}(x)=\sum_{n=1}^{\infty} 2 x\left\{x^{2}+j_{\nu, n}^{2}\right\}^{-1},
$$

where $0<j_{\nu, 1}<j_{\nu, 2}<\cdots<j_{\nu, n}<\cdots$ are the positive zeros of the Bessel function $J_{\nu}(x)$.

Proof of Theorem 1. Consider first the case $\nu>1 / 2$. Since $F_{1}(x, \nu)>0$ for $x \in[0, \infty)$ we set $h(x):=-\ln F_{1}(x, \nu)$. Using the relationship

$$
d\left(x^{\nu} K_{\nu}(x)\right) / d x=-x^{\nu} K_{\nu-1}(x)
$$

[1, (7.11.21)], we find $2 x^{1 / 2} h^{\prime}(x)=1-K_{\nu-1}(\sqrt{x}) / K_{\nu}(\sqrt{x})$. We then apply (2.1) and

$$
x^{-1 / 2}=\frac{2}{\pi} \int_{0}^{\infty} \frac{d t}{x+t^{2}}
$$

to derive the integral representation

$$
\pi h^{\prime}(x)=\int_{0}^{\infty} \frac{1}{x+t^{2}}\left[1-\frac{2(\pi t)^{-1}}{J_{\nu}^{2}(t)+Y_{\nu}^{2}(t)}\right] d t .
$$

The coefficients of $\left(x+t^{2}\right)^{-1}$ in the above integrand is positive since $J_{\nu}^{2}(t)+$ $Y_{\nu}^{2}(t)>2 /(\pi x)$, when $\nu>1 / 2$, Watson [11, §13.74]. This shows that $h^{\prime}$ satisfies (1.2) and $d \omega$ is a positive measure. The same proof works in the case $0<\nu<1 / 2$. The only difference is that $J_{\nu}^{2}(t)+Y_{\nu}^{2}(t)<2 /(\pi x)$ when $0<\nu<1 / 2$. This completes the proof.

Proof of Theorem 2. The proof is similar to that of Theorem 1. When $\nu>$ $1 / 2$ we set $h(x):=-\ln F_{2}(x, \nu)$. Then $d\left(x^{-\nu} I_{\nu}(x)\right) / d x=x^{-\nu} I_{\nu+1}(x),[1$, (7.11.21)], (2.2) and (2.3) yield

$$
h^{\prime}(x)=\frac{1}{2} x^{-1 / 2}-\sum_{n=1}^{\infty}\left(x+j_{\nu, n}^{2}\right)^{-1}=\frac{1}{\pi} \int_{0}^{\infty} \frac{d t}{x+t^{2}}-\sum_{n=1}^{\infty} \frac{1}{x+j_{\nu, n}^{2}} .
$$

The functions $1 /\left(x+t^{2}\right)^{m+1}$ decrease with $t$ for $t>0$ and $m=1,2,3, \ldots$. 
Therefore a discrete approximation to the integral in the above formula implies

$$
\frac{(-1)^{m}}{m !} \frac{d^{m} h^{\prime}(x)}{d x^{m}}>\sum_{n=1}^{\infty} \frac{j_{\nu, n}-j_{\nu, n-1}}{\pi\left(x+j_{\nu, n}^{2}\right)^{m+1}}-\sum_{n=1}^{\infty}\left[x+j_{\nu, n}^{2}\right]^{-m-1},
$$

where $j_{\nu, 0}:=0$. The right hand side in the above inequality is positive when $\nu>1 / 2$ and is negative when $\nu<1 / 2$ since $j_{\nu, n}-j_{\nu, n-1}>\pi$ if $\nu>1 / 2$ but $j_{\nu, n}-j_{\nu, n-1}<\pi$ if $\nu<1 / 2$ [11]. This establishes the complete monotonicity of $h^{\prime}$ when $\nu>1 / 2$. The case $0<\nu<1 / 2$ is similar. To see $h^{\prime}(x)$ cannot be as in (1.2) with $d \omega>0$ note that $\omega$ is unique up to normalization at the points of discontinuity. Furthermore (2.4) gives $d \omega=(2 \pi \sqrt{t})^{-1} d t-d \eta(t)$ where $d \eta(t)$ is a discrete measure with unit masses located at $t=\left(j_{\nu, n}\right)^{2}$, $n=1,2, \ldots$. It is now clear that $d \eta(t)$ is not a positive measure.

\section{AN APPLICATION}

The following integral arose in the analysis of pseudo-differential operators in [13]:

$$
H(t, x)=t\left\{\left(x^{2}+t^{2}\right) / 2\right\}^{-(n+1) / 2} \int_{0}^{\infty} w^{(n-1) / 2} \exp \left\{-w-\frac{m^{2}\left(x^{2}+t^{2}\right)}{4 w}\right\} d w .
$$

The question was to estimate the function $F(\mu, x)$ for $x>0$, and $\mu \leq m$,

$$
F(\mu, x)=\int_{0}^{\infty} e^{\mu t} H(t, x) d t=x \int_{0}^{\infty} e^{\mu x t} H(x t, x) d t .
$$

The main result of this section is Theorem 4.

Theorem 4. The following results hold:

(i) There is a constant $M_{2}$ such that $F(m, x) \geq M_{2} x^{-(n-1) / 2}, x>0$, $n>1$.

(ii) There is a constant $M_{3}$ such that $F(m, x) \leq M_{3} x^{-(n-1) / 2}$, for $x>0$ and $n>2$.

In [13] M. W. Wong proved that there is a constant $C$ independent of $\mu$ such that the inequality $F(\mu, x) \leq C x^{-(n-1) / 2} /(m \sqrt{2}-\mu), x>0, n>1$, holds for $\mu<m$. He then let $\mu \rightarrow m$. Note the difference between the latter result and (ii).

We first prove part (i). Replace $w$ by $w / 2$ in (3.1) and combine the fact $K_{-\nu}(x)=K_{\nu}(x)$ with the integral representation [1, (7.12.24)]

$$
2 K_{\nu}(a x)=a^{\nu} \int_{0}^{\infty} t^{-1-\nu} \exp \left\{-\frac{x}{2}\left(t+a^{2} / t\right)\right\} d t,
$$

to find that

$$
H(t, x)=2 t\left\{m^{2} /\left(x^{2}+t^{2}\right)\right\}^{(n+1) / 4} K_{(n+1) / 2}\left(m\left(x^{2}+t^{2}\right)^{1 / 2}\right) .
$$


In view of the latter equality, (1.4) and (3.2), we obtain

$$
F(m, x) \geq 2^{(n+1) / 2} \Gamma((n+1) / 2) x^{n-1} \int_{0}^{\infty} t\left(1+t^{2}\right)^{-(n+1) / 2} e^{m x t-m x \sqrt{1+t^{2}}} d t
$$

The function $t-\left(1+t^{2}\right)^{1 / 2}$ increases with $t$ on $[0, \infty)$, hence $t-\left(1+t^{2}\right)^{1 / 2} \geq-1$ for $t \geq 0$. Thus the exponential function in (3.4) is bounded below by $e^{-m x}$ and a simple calculation establishes the lower bound

$$
F(m, x) \geq 2^{(n-1) / 2} \Gamma((n-1) / 2) x^{n-1} e^{-m x}, \quad n>1, x>0,
$$

and (i) follows.

To prove (ii) we note that $\sqrt{x} e^{x} K_{\nu}(x) \rightarrow(\sqrt{\pi}) / 2$ as $\dot{x} \rightarrow \infty, \nu>-1 / 2[1$, $\S 7.4]$. The function $x^{\nu} K_{\nu}(x)$ decreases with $x$, is continuous on $(0, \infty)$ and continuous on the right at $x=0$. Therefore there are constants $A, C_{1}$, and $C_{2}$ such that

$$
\begin{array}{r}
\sqrt{t} K_{(n+1) / 2}(t) \leq C_{1} e^{-t}, \quad A \leq t \text { and } t^{(n+1) / 2} K_{(n+1) / 2}(t) \leq C_{2} e^{-t} \\
\text { for } 0 \leq t \leq A .
\end{array}
$$

We substitute (3.3) in the second equality in (3.2) and then express the integral as the sum of two integrals, $l_{1}$ and $l_{2}$. In the first integral $0 \leq$ $m^{2} x^{2}\left(1+t^{2}\right) \leq A^{2}$ while in the second integral $m^{2} x^{2}\left(1+t^{2}\right) \geq A^{2}$. We set $\mu=m$, apply the bounds in (3.6), and then change the limits of integration to 0 and $\infty$ to get

$$
l_{1} \leq 2 C_{2} x^{1-n} \int_{0}^{\infty} t\left(1+t^{2}\right)^{-(n+1) / 2} \exp \left[x m\left(t-\sqrt{1+t^{2}}\right)\right] d t
$$

and

$$
l_{2} \leq 2 C_{2} m^{n / 2} x^{1-n / 2} \int_{0}^{\infty} t\left(1+t^{2}\right)^{-n / 4-1 / 2} \exp \left[m x\left(t-\sqrt{1+t^{2}}\right)\right] d t .
$$

In both integrals we use $\exp \left[m x\left\{t-\left(1+t^{2}\right)^{1 / 2}\right\}\right]<1$ and note that the rest of the integrand is integrable when $n>2$. This proves part (ii).

\section{RoOTS OF TWO TRANSCENDENTAL EQUATIONS}

The proofs of Theorems 3 and 5 use (2.1), (2.2), and the three term recurrence relations

$$
\begin{gathered}
I_{\nu-1}(z)=I_{\nu+1}(z)+\frac{2 \nu}{z} I_{\nu}(z), \\
K_{\nu+1}(z)=K_{\nu-1}(z)+\frac{2 \nu}{z} K_{\nu}(z) .
\end{gathered}
$$

Proof of Theorem 3. From (2.2) and (4.1) we find

$$
\frac{I_{\nu-1}(z)}{I_{\nu}(z)}=\frac{2 \nu}{z}+\sum_{n=1}^{\infty} \frac{2 z}{z^{2}+j_{\nu, n}^{2}}
$$


and (1.6) becomes

$$
b^{3}\left[\frac{\nu}{a \sqrt{z}}+\sum_{n=1}^{\infty} \frac{\sqrt{z} / a}{z+j_{\nu, n}^{2} / a^{2}}\right]=a^{3}\left[\frac{\nu}{b \sqrt{z}}+\sum_{n=1}^{\infty} \frac{\sqrt{z} / b}{z+j_{\nu, n}^{2} / b^{2}}\right],
$$

which can be written in the form

$$
\frac{\nu}{z}\left(b^{4}-a^{4}\right)=\sum_{n=1}^{\infty}\left\{\frac{a^{4}\left(\bar{z}+j_{\nu, n}^{2} / b^{2}\right)}{\left|z+j_{\nu, n}^{2} / b^{2}\right|^{2}}-\frac{b^{4}\left(\bar{z}+j_{\nu, n}^{2} / a^{2}\right)}{\left|z+j_{\nu, n}^{2} / a^{2}\right|^{2}}\right\} .
$$

Set

$$
z=\xi+i \eta
$$

We first show that $\xi<0$. To do so we equate the real parts of (4.3) to get

$$
\frac{\xi \nu\left(b^{4}-a^{4}\right)}{\xi^{2}+\eta^{2}}=\sum_{n=1}^{\infty} \frac{A_{n}+\eta^{2} B_{n}}{\left|\left(z+j_{\nu, n}^{2} / a^{2}\right)\left(z+j_{\nu, n}^{2} / b^{2}\right)\right|^{2}},
$$

where

$$
\begin{aligned}
& B_{n}:=a^{4}\left(\xi+j_{\nu, n}^{2} / b^{2}\right)-b^{4}\left(\xi+j_{\nu, n}^{2} / a^{2}\right), \\
& A_{n}:=\left(\xi+j_{\nu, n}^{2} / a^{2}\right)\left(\xi+j_{\nu, n}^{2} / b^{2}\right)\left[a^{4}\left(\xi+j_{\nu, n}^{2} / a^{2}\right)-b^{4}\left(\xi+j_{\nu, n}^{2} / b^{2}\right)\right] .
\end{aligned}
$$

When $\xi \geq 0$ it is easy to see that

$$
\frac{A_{n}}{a^{2}-b^{2}}>0 \text { and } \frac{B_{n}}{a^{2}-b^{2}}>0 .
$$

If we divide both sides of (4.4) by $a^{2}-b^{2}$, we see that when $\xi \geq 0$ the new left side is less than or equal to zero while the new right hand side is positive. This contradiction shows that $\xi \leq 0$. In particular (1.6) has no positive roots.

It only remains to show that there is a positive integer $k$ such that the circle (1.7) contains all roots of (1.6) in its interior. Multiply (4.3) by $z$ and then equate the imaginary parts of both sides of the resulting equation. When $\eta \neq 0$ we obtain

$$
\sum_{n=1}^{\infty} \frac{a^{6} j_{\nu, n}^{2}}{\left|z+j_{\nu, n}^{2} / b^{2}\right|^{2}}=\sum_{n=1}^{\infty} \frac{b^{6} j_{\nu, n}^{2}}{\left|z+j_{\nu, n}^{2} / a^{2}\right|^{2}} .
$$

This shows that the quantity

$$
a^{2}\left|a^{2} z+j_{\nu, n}^{2}\right|^{2}-b^{2}\left|b^{2} z+j_{\nu, n}^{2}\right|^{2}
$$

cannot have the same sign for all $n, n \geq 1$. The quantity in (4.5) is

$$
\left(a^{6}-b^{6}\right)\left(\xi^{2}+\eta^{2}\right)+j_{\nu, n}^{4}\left(a^{2}-b^{2}\right)+2 \xi j_{\nu, n}^{2}\left(a^{4}-b^{4}\right),
$$

which is a constant multiple of

$$
\left(a^{4}+b^{4}+a^{2} b^{2}\right)\left(\xi^{2}+\eta^{2}\right)+j_{\nu, n}^{4}+2 \xi j_{\nu, n}^{2}\left(a^{2}+b^{2}\right) .
$$

Thus the above expression must change sign as $n$ increases from 1 to $\infty$. Since $j_{\nu, n} \rightarrow \infty$ as $n \rightarrow \infty$, the expression (4.6) will be positive eventually, 
hence it must be negative for a finite value of $n$, say $n=k$. This shows that all complex zeros are in the interior of the circle (1.7).

Finally we show that (1.6) has infinitely many real roots. If $z<0$ then $I_{\nu}(\sqrt{z})$ is a constant multiple of $J_{\nu}\left((-z)^{1 / 2}\right)$ and $J_{\nu}(z)$ has infinitely many zeros and they are all real when $\nu>-1$. Thus when $z=-x$ the graphs of the right and left hand sides of (1.6) resemble the graphs of $a^{3} \cot (b \sqrt{x})$ and $b^{3} \cot (a \sqrt{x})$, respectively. It is then clear that there are infinitely many negative roots of (1.6) when $\nu>0$. This completes the proof of Theorem 3.

Theorem 5. Let $a>0, b>0, \nu>0, a \neq b$. Then the nonreal roots of

$$
b^{3} K_{\nu+1}(a \sqrt{z}) / K_{\nu}(a \sqrt{z})=a^{3} K_{\nu+1}(b \sqrt{z}) / K_{\nu}(b \sqrt{z}), \quad z \neq 0,
$$

lie in the sector

$$
|\eta / \xi|<a b / \sqrt{a^{4}+b^{4}+a^{2} b^{2}}, \quad \xi=\operatorname{Re} z, \eta=\operatorname{Im} z .
$$

Proof. We apply (2.1) and (4.2) to express the transcendental equation in Theorem 5 in the form

$$
\int_{0}^{\infty} \frac{t^{-1}}{J_{\nu}^{2}(t)+Y_{\nu}^{2}(t)}\left\{\frac{a^{4} b^{2}}{b^{2} z+t^{2}}-\frac{b^{4} a^{2}}{a^{2} z+t^{2}}\right\} d t=\frac{\nu \pi^{2}}{2 z}\left(b^{4}-a^{4}\right) .
$$

The rest of the proof is similar to the proof of Theorem 3. Write

$$
z=\xi+i \eta
$$

and equate real parts of both sides of (4.7). The real part of the quantity in the curled brackets in (4.7) is

$$
\left[D_{n}+\eta^{2} C_{n}\right] /\left|\left(z+t^{2} / a^{2}\right)\left(z+t^{2} / b^{2}\right)\right|^{2},
$$

where

$$
\begin{aligned}
& C_{n}=\left(a^{4}-b^{4}\right) \xi+\frac{t^{2}\left(a^{6}-b^{6}\right)}{a^{2} b^{2}}, \\
& D_{n}=\left(\xi+t^{2} / a^{2}\right)\left(\xi+t^{2} / b^{2}\right)\left[a^{2}\left(a^{2} \xi+t^{2}\right)-b^{2}\left(b^{2} \xi+t^{2}\right)\right] .
\end{aligned}
$$

When $\xi \geq 0$ it follows that both $C_{n} /\left(a^{2}-b^{2}\right)$ and $D_{n} /\left(a^{2}-b^{2}\right)$ are positive and this leads to a contradiction as in the proof of Theorem 3. Therefore $\xi=\operatorname{Re} z<0$. When $\eta=\operatorname{Im} z \neq 0$, multiply (4.7) by $z$ and then equate the imaginary parts of the resulting equation to see that as $t$ varies from 0 to $\infty$ the function

$$
a^{2}\left|a^{2} z+t^{2}\right|^{2}-b^{2}\left|b^{2} z+t^{2}\right|^{2}
$$

must change sign at least once. The function in (4.8) is a constant multiple of

$$
\left(a^{4}+b^{4}+a^{2} b^{2}\right)\left(\xi^{2}+\eta^{2}\right)+t^{4}+2 \xi t^{2}\left(a^{2}+b^{2}\right),
$$

that is

$$
\left(a^{4}+b^{4}+a^{2} b^{2}\right) \eta^{2}-a^{2} b^{2} \xi^{2}+\left[t^{2}+\xi\left(a^{2}+b^{2}\right)\right]^{2} .
$$


In order for the above function of $t$ to change sign on $(0, \infty)$ we must have

$$
\left(a^{4}+b^{4}+a^{2} b^{2}\right) \eta^{2}-a^{2} b^{2} \xi^{2}<0,
$$

and Theorem 5 follows.

\section{ACKNOWLEDGMENT}

Part of this work was motivated by a lecture Dr. Man Wah Wong gave at a seminar at York University. I am very grateful to Man Wah for bringing his work [13] to my attention. I thank Martin Muldoon for hospitality during my visit to York University and for very stimulating discussions during the preparation of this note. I also thank Dr. H. Hattori for asking me to confirm his conjecture. Thanks to the referee for correcting several typographical errors in the manuscript.

\section{REFERENCES}

1. A. Erdelyi, W. Magnus, F. Oberhettinger and F. Tricomi, Higher transcendental functions, volume 2, McGraw-Hill, New York, 1954.

2. W. Feller, An introduction to probability theory and its applications, Vol. 2, John Wiley, New York, 1966.

3. E. Grosswald, The student t-distribution of any degrees of freedom is infinitely divisible, $\mathrm{Z}$. Wahrscheinlichkeitstheorie und verw. Gebiete 36 (1976), 103-109.

4. P. Hartman, Difference equations, disconjugacy, principal solutions, Green's functions, complete monotonicity, Trans. Amer. Math. Soc. 246 (1978), 1-30.

5. __ Uniqueness of principal values, complete monotonicity of logarithmic derivatives of principal solutions, and oscillation theorems, Math. Ann. 241 (1979), 257-281.

6. H. Hattori, private communication.

7. M. E. H. Ismail, Bessel functions and the infinite divisibility of the student t-distribution, Ann. Prob. 5 (1977), 582-585.

8. M. E. H. Ismail and D. H. Kelker, Special functions, Stieltjes transforms and infinite divisibility, SIAM J. Math. Anal. 10 (1979), 884-901.

9. J. Pitman and M. Yor, Bessel processes and infinitly divisible laws, in Stochastic Integrals (Proc. Sympos. Univ. Durham, Durham 1980), Lecture Notes in Math. No. 851, Springer-Verlag, Berlin, 1981, 285-370.

10. O. Thorin, On the infinite divisibility of the Pareto distribution, Scad. Actuar. J. (1977), No. 1, 31-40.

11. G. N. Watson, A treatise on the theory of Bessel functions, second edition, Cambridge University Press, Cambridge, 1944.

12. D. V. Widder, The Laplace transform, Princeton University Press, Princeton, 1941.

13. M. W. Wong, On eigenvalues of pseudo-differential operators, Bull. London Math. Soc. 19 (1987), 63-66.

Department of Mathematics, University of South Florida, Tampa, Florida, 33620 